\title{
Marquer le nombre du nom et de l'adjectif : une difficulté persistante dans deux corpus de dictées d'élèves avancés
}

\author{
Hélène Le Levier ${ }^{11}$, et Catherine Brissaud ${ }^{1}$ \\ ${ }^{1}$ Université Grenoble Alpes, Lidilem, F-38000, France
}

\begin{abstract}
Résumé. Respecter les marques du pluriel attendues sur les noms et les adjectifs est une difficulté du français, largement documentée à l'école primaire. Nous avons pu vérifier que cette difficulté se maintenait au cours du secondaire et jusque dans le supérieur à travers deux corpus de dictées recueillis auprès d'élèves français de la quatrième année du secondaire et de la première année de l'enseignement supérieur technique. Dans l'ensemble, ces élèves omettent significativement plus de marques au pluriel qu'ils n'en ajoutent au singulier. Ces résultats ne sont cependant pas homogènes. Le pluriel de l'adjectif est moins réussi que celui du nom. Les caractéristiques lexicales et syntaxiques de chaque forme influencent la réussite du marquage en nombre. D'un point de vue didactique, ces résultats invitent à prendre en compte les multiples facteurs qui influencent le marquage en nombre dans l'accompagnement des élèves tout au long de leur scolarité.
\end{abstract}

\begin{abstract}
Marking the grammatical number of the noun and the adjective : a persistent difficulty in two collections of advanced pupils dictations. The difficulty of French inflection for the noun and the adjective is well documented for primary school pupils. We confirm that this difficulty remains among secundary and superior studies through two collections of dictations from french pupils of forth year of secundary school and first year of technical superior studies. These students neglect to mark the plural significantly more often than they add a false singular mark. These results are not homogeneous. The adjective plural is less successful than the noun plural. Lexical and syntactic features affect the success of plural marking. From a didactic point of view, these results invite us to take into account the multiple factors which have an effect on number marking all through written French teaching process.
\end{abstract}

La demande sociale en termes de maitrise du code orthographique est très forte de nos jours. Dans le même temps, plusieurs études ont montré un recul des compétences orthographiques des élèves français à âge égal, d'une part de 11 à 15 ans (Manesse et Cogis, 2007), d'autre part à 11 ans, en fin de scolarité primaire (Andreu \& Steinmetz, 2016). Ces études ont par ailleurs montré que le recul des compétences orthographiques affectait surtout l'orthographe grammaticale, en particulier la morphographie du verbe et de l'adjectif et que l'homophonie verbo-nominale de formes distinguées à l'oral pouvait poser problème.Le marquage du pluriel est par ailleurs identifié dès les premières années d'apprentissage comme une difficulté du français écrit (Totereau, Thévenin et Fayol, 1997 ; Lefrançois, 2009 ; Fayol et Got, 1991). Mais les études consacrées aux compétences orthographiques des francophones avancés,

1 Corresponding author : helene-fleur.le-levier@ac-lille.fr 
scolarisés dans le secondaire et au-delà, restent rares, de sorte qu'on ne sait pas dans quelle mesure le marquage du pluriel pose durablement problème. On peut donc se demander où se situent les difficultés orthographiques des élèves ayant suivi une scolarité en France. Pour apporter des éléments de réponse à cette question, nous nous appuierons sur deux corpus de dictées recueillies auprès d'élèves scolarisés en France à des niveaux avancés : un corpus de 735 dictées d'élèves de troisième (4ème année du secondaire) de l'académie de Grenoble recueilli en 2016 et un corpus de 178 dictées d'élèves de sections de techniciens de supérieur (premier cycle de l'enseignement supérieur technique) de l'académie de Lille recueilli en 2018. Nous reviendrons d'abord sur ce que des corpus de dictées peuvent nous apprendre de l'accord en nombre du nom et de l'adjectif. Nous expliquerons ensuite notre méthodologie et le corpus qu'elle nous a permis de construire, avant de présenter nos principaux résultats et de les discuter.

\section{L'accord en nombre : une zone sensible de l'orthographe française}

L'orthographe du français est peu transparente, cela signifie en particulier qu'il est difficile de deviner sa forme graphique à partir de sa forme sonore (Fayol \& Jaffré, 2014). Le français présente par ailleurs un système morphographique très riche à l'écrit dont beaucoup de formes sont homophones. Cette caractéristique participe à l'opacité du français et implique des processus d'apprentissage différents. En effet, là où la reconnaissance d'homophones lexicaux repose sur le stockage de formes en mémoire, la discrimination des homophones grammaticaux exige nécessairement une analyse morphosyntaxique. Ce point n'implique cependant pas que la mémoire ne joue aucun rôle dans la production de formes fléchies. Plusieurs études ont ainsi montré la possible influence de la fréquence des formes rencontrées en lecture sur la production de formes exigeant une analyse morphographique, notamment les formes en [E] (Brissaud, Chevrot, \& Lefrançois, 2006, Fayol \& Pacton, 2006) et les formes verbales séparées de leur sujet par un pronom (Largy, Fayol \& Lemaire, 1996).La forme timbres s'impose facilement dans Ils les timbre(s), le nom timbre étant plus fréquent que le verbe timbrer. Mais ce rôle de la mémoire contribue précisément à produire des formes qui ne sont pas conformes à la norme orthographique définie institutionnellement. Ce phénomène montre que l'identification des zones qui posent problème aux francophones ne va pas de soi : un même phénomène peut poser peu de problèmes aux scripteurs dans certaines configurations syntaxiques ou lexicales et beaucoup plus dans d'autres du fait de la complexité des processus à l'œuvre dans la production des formes graphiques. Brissaud (2015) constate ainsi dans un corpus de dictées recueilli auprès d'élèves de troisième que la marque du pluriel dans le syntagme des arbres est beaucoup moins souvent omise ( 2 omissions sur 98 occurrences) que dans le syntagme couverte d'arbres (38 omissions sur 98 occurrences). Le contexte syntaxique influence donc la production de la marque de nombre attendue.

Nous allons exposer ici plusieurs phénomènes identifiés par la recherche qui ont guidé la construction des deux dictées utilisées dans cette étude, en nous concentrant sur les éléments liés à l'accord en nombre.

L'accord en nombre est identifié comme une difficulté qui apparait tôt dans le développement des capacités scripturales et se maintient longtemps. En effet, les marques d'accord au pluriel muettes peuvent prendre des formes diverses, principalement $-s$ dans le système nominal et -ent dans le système verbal. Totereau, Thévenin et Fayol $(1997, \mathrm{p} ; 101$ et suivantes) ont montré que des élèves des trois premières années de l'élémentaire acquéraient le pluriel de façon plus progressive, plus facilement sur le nom que sur l'adjectif et le verbe et plus précocément en compréhension qu'en production. Lefrançois (2009) a mis en évidence que, si la maitrise du pluriel progresse entre la première et la cinquième année du primaire, 
elle n'est pas stabilisée en fin de cinquième année notamment du fait de la survivance de procédures sémantiques plus souvent sources d'erreurs que les procédure morphosyntaxiques. Un syntagme comme tout le monde va ainsi entrainer une surproduction de marques du pluriel sur le nom du fait de la notion de pluralité liée à son sens.

Les études menées auprès de scripteurs plus âgés sont rares. Pach, Jacquemin, Millet et Billiez (1994) analysent les écarts à la norme orthographique dans des corpus d'écrits d'étudiants en situation formelle d'examen et informelle de prise de notes et de lycéens professionnels en situation formelle scolaire. L'omission des marques de nombre sur les noms et les adjectifs apparait en tête de la liste des «neufs façons favorites d'enfreindre la loi » (p. 91). Elle est fortement représentée dans tous les corpus même s'ils notent qu'elle est beaucoup moins dense chez les étudiants en situation formelle que chez les lycéens en situation formelle. Cette étude confirme donc la persistance de difficultés à marquer le nombre des noms et plus encore des adjectifs (p. 64) chez des scripteurs avancés. On retrouve ce phénomène dans l'étude consacrée par Blondel, Brissaud et Rinck (2016) au pratiques orthographiques de scripteurs ordinaires et de scripteurs en difficulté avec l'écrit. Elles montrent que l'omission des marques du pluriel est massive chez les scripteurs adultes en difficulté avec l'écrit. Elle est moins marquée chez les scripteurs ordinaires mais subsiste néanmoins dans un syntagme comme une boite d'allumettes où le pluriel n'est pas marqué à l'oral. Fayol et Got (1991) montrent quant à eux que la nature de ces difficultés d'accord peut changer au cours du temps. Ils ont ainsi mis au jour une tendance à l'accord de proximité plus marquée chez des scripteurs adultes que chez des enfants d'âge primaire. Les adultes ayant participé à l'étude étaient davantage influencés que les enfants par la présence d'un complément entre le sujet et le verbe dans une phrase du type Le père des enfants danse et étaient plus enclins à produire dansent dans ce contexte. L'accord en nombre apparait donc comme une difficulté récurrente en français mais dont l'intensité est variable suivant les configurations syntaxiques.

Des études ont par ailleurs montré que les difficultés d'identification des marques correctes d'accord pouvaient être liées à un conflit entre accord sémantique et accord syntaxique. Cogis (2005) évoque ainsi des cas d'ajout de $e$ à une forme verbale qui n'en exige pas du fait de l'identité féminine du scripteur, produisant des énoncés du type " j'ai écrit joue avec un $e$ parce que je suis une fille ». L'élève va alors chercher dans la situation d'énonciation la motivation d'un écart dont la source devrait être cherchée dans la syntaxe de la phrase qu'il écrit. Cette difficulté à laisser de côté la référence sémantique au réel pour se concentrer sur le fonctionnement syntaxique explique pour Cogis (2007) la difficulté que représente l'accord de l'adjectif par rapport à l'accord du nom. En effet, un nom au pluriel désigne une pluralité alors que ce n'est pas le cas d'un adjectif au pluriel. Dans le syntagme les petits chats, le sens du mot chat est modifié par l'ajout du $s$ qui signifie la pluralité (" ils sont plusieurs » disent les élèves), mais le $s$ au bout de petits ne modifie pas le sens de l'adjectif, il ne fait que confirmer son lien avec le mot chat. L'accord de l'adjectif appelle donc des analyses purement syntaxiques, plus abstraites et donc potentiellement plus difficiles à mettre en œuvre que l'accord du nom.

Compte tenu des études assez nombreuses sur l'enseignement primaire et des quelques travaux concernant le secondaire et le supérieur, on peut s'attendre à retrouver chez les élèves de troisième et de STS que nous avons interrogés certaines difficultés identifiées. Nous faisons l'hypothèse que la marque du pluriel sera plus souvent omise quand elle est attendue qu'ajoutée quand la cible est au singulier et que les marques du nombre seront plus souvent réussies sur les formes nominales que sur les formes adjectivales. Par ailleurs, il serait attendu que la que réussite des formes adjectivales et nominales ne soit pas homogène entre les formes de la dictée mais varie en fonction de leurs configurations lexicales et syntaxiques. 


\section{Deux corpus de dictées}

\subsection{Le choix de la dictée comme mode de recueil de la production orthographique}

La dictée est, depuis près de deux siècles, l'exercice roi de l'enseignement de l'orthographe en France (Chervel, 2008). Comme outil pédagogique, elle est souvent remise en cause mais aussi repensée pour en faire un outil de formation et non simplement d'évaluation. En recherche, on lui préfère souvent des méthodologies permettant de contrôler plus étroitement les caractéristiques lexicales et syntaxiques de chaque item testé : des listes de phrases lacunaires dont les enquêtés doivent graphier les formes testées (Brissaud et Chevrot, 2011); parfois accompagnées de questionnaires à choix multiples (David, Guyon, et Brissaud, 2006), des items à corriger (Largy \& Dédéyan, 2002) ou de courtes phrases dictées (Fayol et Pacton, 2006). Cependant ces méthodologies présentent l'inconvénient de proposer aux participants des situations d'écriture peu complexes qui ne les amènent pas à gérer simultanément toutes les difficultés de phrases complètes et complexes. D'autres méthodologies cherchent au contraire à recueillir des écrits qui n'ont pas été construits spécifiquement pour l'enquête et révèlent donc davantage les caractéristiques de l'écriture de l'enquêté en situation réelle (Pach, Jacquemin, Millet et Billiez, 1994 ; Voiriot-Cordary, 2005 ; Mout, 2013). Cependant, ces méthodologies créent des corpus difficiles à exploiter, notamment parce que les enquêtés ne produisent pas nécessairement les mêmes types de forme d'un écrit à l'autre.

La dictée de texte constitue donc une forme de compromis qui permet à la fois de recueillir les mêmes formes chez un grand nombre d'enquêtés et de tester les capacités de ces enquêtés à respecter la norme orthographique dans une situation d'écriture relativement complexe. Pour autant, si la dictée est rapide à recueillir, elle est longue à dépouiller (Trosseille et Brissaud, 2015) et il est important de contrôler le plus étroitement possible ce que cette dictée mesure. En effet, comme l'ont montré Dister et Moreau (2019) à partir d'un corpus de dictées de concours d'orthographe pour enfants en Belgique, une dictée n'évalue pas le niveau global des élèves. Elle évalue les difficultés que pose le texte choisi.

L'un des objectifs des deux corpus de dictées que nous avons recueillis était de tester la capacité des élèves à marquer le pluriel. Elles ont par ailleurs été accompagnées d'entretiens métagraphiques qui ciblaient notamment des formes adjectivales au pluriel. Nous allons présenter ici les résultats obtenus sur les formes nominales et adjectivales.

\subsection{La dictée de troisième}

Le premier corpus que nous allons exploiter ici est composé des dictées écrites par 735 élèves de quatrième année du secondaire de l'académie de Grenoble en 2016. Le texte de la dictée a été élaboré à partir du test Repérage orthographique collectif (ROC) établi par Michel Zorman et ses collaborateurs et modifié par des linguistes pour tester l'expression du nombre et l'accord du participe passé chez les collégiens participant à l'enquête. Les formes nominales sont signalées en gras et les formes adjectivales en italique.

Je vais vous raconter l'histoire d'un gentil petit garçon qui s'appelle Jo. Il habite chez son oncle, un vieux monsieur. Ils vivent dans une cité. Cet enfant possède un don extraordinaire. En effet, ses yeux verts voient beaucoup plus loin et plus précisément que ceux de tout le monde! Dans ses pupilles qui s'agrandissent et qui brillent, on devine des jumelles microscopiques perfectionnées. Dans la ville peuplée 
d'immeubles bariolés, personne n'est informé de ce don sauf son oncle qui l'a toujours considéré comme son fils. Alors, souvent, Jo et et le vieil homme vont regarder ensemble par la fenêtre et Jo décrit ce qu'il voit.

Tableau 1: Noms et adjectifs de la dictée de troisième

\begin{tabular}{|l|l|l|}
\hline Nature grammaticale & $\begin{array}{l}\text { Genre, nombre ou } \\
\text { personne }\end{array}$ & Mots concernés \\
\hline \multirow{2}{*}{ Noms } & Au singulier & $\begin{array}{l}\text { histoire, garçon, oncle, monsieur, cité, enfant, don, monde, } \\
\text { ville, don, oncle, fils, homme, fenêtre }\end{array}$ \\
\cline { 2 - 3 } & Au pluriel & yeux, pupilles, jumelles, immeubles \\
\hline Adjectifs & Masculin singulier & gentil, petit, vieux, extraordinaire, vieil, informé \\
\cline { 2 - 3 } & Masculin pluriel & verts, bariolés \\
\cline { 2 - 3 } & Féminin singulier & peuplée \\
\cline { 2 - 3 } & Féminin pluriel & microscopiques \\
\hline
\end{tabular}

\subsection{La dictée utilisée auprès des étudiants de sections de techniciens supérieurs}

Cette dictée s'est inspirée de la dictée utilisée en troisième mais son contenu a été adapté à l'âge des 178 étudiants qui l'ont passée. Le choix de deux jeunes filles comme personnages principaux a permis de tester l'accord en genre et en nombre. Les formes nominales sont signalées en gras et les formes adjectivales en italique.

Je vais vous parler de deux étudiantes en commerce international parties étudier dans un pays éloigné. Elles ont préparé leur voyage avec beaucoup de soin et chacune a organisé ses affaires pour être prête le 20 aout. Ces deux jeunes filles ne manquaient pas d'amis/ ies : elles ont consacré un weekend entier à faire leurs adieux à tout le monde. Le jour du départ, elles ont enfilé les vêtements qu'elles avaient choisis la veille et vérifié la liste de papiers règlementaires qu'elles avaient soigneusement préparée / és. Ces préparatifs achevés, la première s'est dépêchée de saluer une dernière fois ses parents, tandis que la seconde goutait ces tout derniers instants avant l'aventure. Quel évènement !

Tableau 2: Noms et adjectifs de la dictée de STS

\begin{tabular}{|l|l|l|}
\hline Nature grammaticale & Genre, nombre ou personne & Mots concernés \\
\hline \multirow{2}{*}{ Noms } & Au singulier & $\begin{array}{l}\text { commerce, pays, voyage, soin, aout, weekend, monde, jour, } \\
\text { départ, veille, liste, première, seconde, aventure, évènement }\end{array}$ \\
\cline { 2 - 3 } & Au pluriel & $\begin{array}{l}\text { étudiantes, affaires, filles, amis, adieux, vêtements, papiers, } \\
\text { préparatifs, parents, instants }\end{array}$ \\
\hline \multirow{2}{*}{ Adjectifs } & Masculin singulier & éloigné, entier, international \\
\cline { 2 - 3 } & Masculin pluriel & règlementaires, achevés, derniers \\
\cline { 2 - 3 } & Féminin singulier & prête, dernière \\
\cline { 2 - 3 } & Féminin pluriel & parties, jeunes \\
\hline
\end{tabular}




\section{Résultats}

\subsection{Formes nominales}

Tableau 3: Pourcentage de réussite nom par nom dans la dictée de troisième et la dictée de SES.

\begin{tabular}{|c|c|c|c|c|c|}
\hline \multicolumn{3}{|c|}{ Dictée de troisième } & \multicolumn{3}{|c|}{ Dictée de STS } \\
\hline Forme dictée & $\begin{array}{l}\text { Pourcentage } \\
\text { de réussite } \\
\text { mot entier }\end{array}$ & $\begin{array}{l}\text { Pourcentage de } \\
\text { réussite marquage } \\
\text { en nombre }\end{array}$ & $\begin{array}{l}\text { Forme } \\
\text { dictée }\end{array}$ & $\begin{array}{l}\text { Pourcentage de } \\
\text { réussite mot } \\
\text { entier }\end{array}$ & $\begin{array}{l}\text { Pourcentage de réussite } \\
\text { marquage en nombre }\end{array}$ \\
\hline immeubles & $47,48 \%$ & $57,48 \%$ & instants & $73 \%$ & $74 \%$ \\
\hline pupilles & $78,50 \%$ & $96,45 \%$ & soin & $74 \%$ & $74 \%$ \\
\hline don & $80 \%$ & $96,49 \%$ & adieux & $79 \%$ & $79 \%$ \\
\hline jumelles & $87,35 \%$ & $90,44 \%$ & préparatifs & $79 \%$ & $93 \%$ \\
\hline garçon & $95,92 \%$ & $97,01 \%$ & parents & $95 \%$ & $95 \%$ \\
\hline histoire & $97,01 \%$ & $98,23 \%$ & vêtements & $84 \%$ & $96 \%$ \\
\hline monde & $97,82 \%$ & $99,04 \%$ & papiers & $96 \%$ & $96 \%$ \\
\hline monsieur & $86,67 \%$ & $99,50 \%$ & filles & $96 \%$ & $96 \%$ \\
\hline cité & $80,82 \%$ & $99,73 \%$ & voyage & $97 \%$ & $97 \%$ \\
\hline yeux & $97,55 \%$ & $99,73 \%$ & monde & $97 \%$ & $97 \%$ \\
\hline oncle & $98,57 \%$ & $99,79 \%$ & évènement & $70 \%$ & $97 \%$ \\
\hline fenêtre & $85,99 \%$ & $99,86 \%$ & amis/ies & $98 \%$ & $98 \%$ \\
\hline ville & $99,05 \%$ & $99,86 \%$ & affaires & $98 \%$ & $98 \%$ \\
\hline enfant & $97,28 \%$ & $100 \%$ & étudiantes & $94 \%$ & $98 \%$ \\
\hline fils & $97,55 \%$ & $100 \%$ & commerce & $99 \%$ & $99 \%$ \\
\hline \multirow[t]{11}{*}{ homme } & $97,69 \%$ & $100 \%$ & aout & $98 \%$ & $99 \%$ \\
\hline & & & weekend & $91 \%$ & $99 \%$ \\
\hline & & & liste & $99 \%$ & $99 \%$ \\
\hline & & & pays & $99 \%$ & $100 \%$ \\
\hline & & & fois & $99 \%$ & $100 \%$ \\
\hline & & & jour & $100 \%$ & $100 \%$ \\
\hline & & & départ & $95 \%$ & $100 \%$ \\
\hline & & & veille & $99 \%$ & $100 \%$ \\
\hline & & & première & $92 \%$ & $100 \%$ \\
\hline & & & seconde & $98 \%$ & $100 \%$ \\
\hline & & & aventure & $96 \%$ & $100 \%$ \\
\hline $\begin{array}{l}\text { Total noms } \\
\text { singuliers }\end{array}$ & $92 \%$ & $98,99 \%$ & $\begin{array}{l}\text { Total noms } \\
\text { singuliers }\end{array}$ & $94 \%$ & $97,6 \%$ \\
\hline $\begin{array}{l}\text { Total noms } \\
\text { pluriels }\end{array}$ & $78 \%$ & $86,06 \%$ & $\begin{array}{l}\text { Total noms } \\
\text { pluriels }\end{array}$ & $89 \%$ & $92,1 \%$ \\
\hline $\begin{array}{l}\text { Toutes } \\
\text { formes }\end{array}$ & $89 \%$ & $96,11 \%$ & $\begin{array}{l}\text { Toutes } \\
\text { formes }\end{array}$ & $92 \%$ & $95,5 \%$ \\
\hline
\end{tabular}


Le tableau 3 présente l'ensemble des formes nominales des deux dictées classées de la moins réussie à la mieux réussie en ce qui concerne la marque du nombre. La colonne "pourcentage de réussite marquage du nombre » dénombre les formes qui présentent la marque de nombre attendu (singulier ou pluriel) compte non tenu des erreurs sur la base. Nous avons signalé en gras les mots pour lesquels l'écart entre la réussite du mot entier et la réussite de la marque de nombre est égale à $10 \%$ ou plus du total des formes recueillies. Il s'agit donc des mots qui concentrent les plus grandes difficultés lexicales. En STS, il s'agit de trois mots comportant des accents : préparatifs, vêtements et évènement. L'examen des formes produites montre que la totalité des erreurs lexicales commises sur préparatifs et vêtements sont des omissions ou substitutions d'accent, de même que la grande majorité des erreurs sur évènement, qui compte également quelques doublements du $n$. Ce constat rejoint celui de Pach, Jacquemin, Millet et Billiez (1994) qui analysent un corpus d'écrits de scripteurs avancés et montrent que les variations sur les diacritiques représente les quatre cinquièmes du total. Dans la dictée de troisième, on retrouve ce phénomène à travers le mot fenêtre dont l'essentiel des variations lexicales est lié à la graphie du $[\varepsilon]$ soit par substitution de l'accent, soit par ajout d'un consonne double (fenêttre) ou d'un digramme (feneitre). On retrouve quelques erreurs d'accent sur cité, mais la majorité des écarts lexicaux consiste en l'ajout d'un $e$ marquant très probablement le féminin (citée) choisi par 13,64 \% des élèves. Les autres noms concernés comportent des géminées, immeubles et pupilles, ou un homophone courant pour don, orthographié dont dans $13,91 \%$ des cas. Enfin, monsieur donne lieu à de très nombreuses variantes mais la grande majorité d'entre elles substitue un $e$ à on pour graphier le [ə] du début du mot. De fait, il s'agit là d'une graphie très irrégulière à l'échelle du système phonographique du français.

$\mathrm{Si}$ on se concentre sur les marques du nombre, dont la réussite n'est pas liée à la connaissance lexicale du mot mais au traitement des relations syntaxiques entre les éléments de la phrase, on constate que les noms au singulier sont mieux réussis que les noms au pluriel, ce qui n'est pas étonnant puisque le singulier correspond massivement en français à une absence de marque. Étant donné qu'il n'est pas raisonnable de considérer que tous les élèves ont les mêmes chances de réussite, la significativité de l'écart entre la réussite moyenne des formes au singulier et des formes au pluriel ne peut être évaluée par un test de comparaison d'échantillons identiquement distribués. Nous avons construit un test prenant en compte la variabilité des élèves et qui permet de vérifier que cet écart est significatif (voir annexe). Par ailleurs, la représentation graphique de la densité d'erreurs par élève au singulier et au pluriel confirme à la fois la diversité des profils d'élèves et la plus grande difficulté du pluriel. 


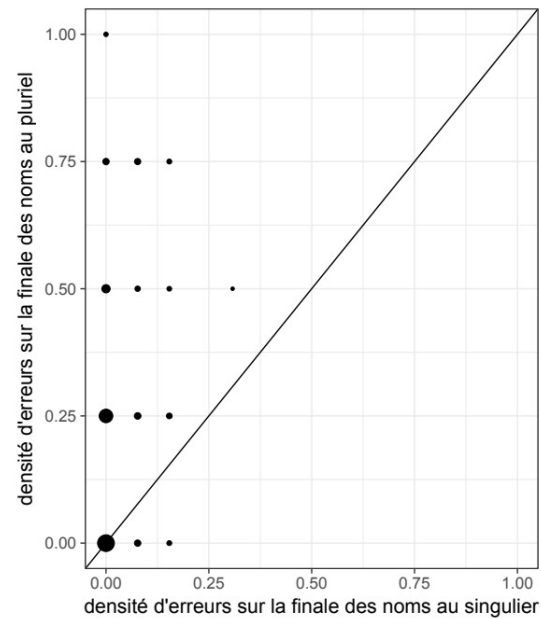

Illustration 1: Densité d'erreurs sur la finale des noms au singulier (abscisse) et au pluriel (ordonnée) dans la dictée de troisième.

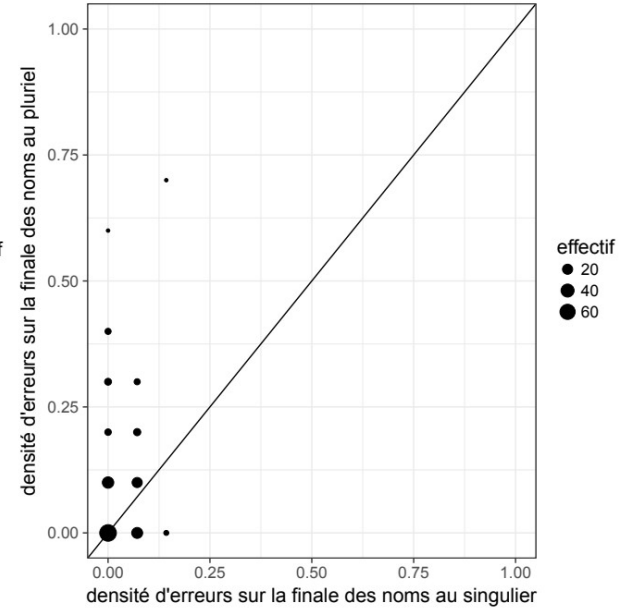

Illustration 2: Densité d'erreurs sur la finale des noms au singulier (abscisse) et au pluriel (ordonnée) dans la dictée de STS.

Les illustrations 1 et 2 représentent la répartition des densités d'erreurs sur les marques de nombre des noms au singulier et au pluriel par élève dans les deux corpus de dictées. Si le nombre d'erreurs de marque était sensiblement égal, ils se répartirait le long de la ligne tracée au milieu. Ces représentations graphiques illustrent donc le déséquilibre entre réussite au pluriel et réussite au singulier. On constate dans les deux représentations que le point concentrant le plus grand nombre d'élèves est le point $(0,0)$ où se situent les élèves qui n'ont commis aucune erreur de marquage de nombre. Pour autant, ces points ne représentent pas la majorité des élèves. Sur les deux graphiques, on trouve également un petit nombre d'élèves ayant commis des erreurs sur des noms au singulier mais pas au pluriel (points sur la ligne verticale correspondant au 0 ). Il est néanmoins très net que les élèves commettant des erreurs au pluriel et pas au singulier sont plus nombreux. Sur le graphique correspondant aux élèves de troisième, on voit même apparaitre des élèves n'ayant marqué aucune forme au pluriel. Ceux-ci sont cependant minoritaires. S'il est vrai que marquer le pluriel des noms semble encore poser problème à une grande partie des scripteurs avancés auprès desquels nous avons enquêté, ils sont très majoritaires dans les deux enquêtes à marquer le pluriel sur au moins une partie des formes qui l'exigent.

$\mathrm{Si}$ on se reporte au tableau 3, on constate cependant que certaines formes au pluriel concentrent des omissions de marquage du nombre plus importantes que les autres. Dans la dictée de troisième, il s'agit du mot immeubles dont la marque de pluriel est notée dans moins de $60 \%$ des cas. Ce phénomène peut s'expliquer par le contexte syntaxique dans lequel est inséré le mot. En effet, le syntagme dans la ville peuplée d'immeubles ne fait pas apparaitre de déterminant dont le pluriel serait sensible à l'oral. De fait, des entretiens métagraphiques menés avec une partie des élèves ayant passé la dictée ont permis d'établir que la grande majorité de ceux ayant omis la marque de pluriel la rajoutent lors de l'entretien, suite au simple fait de pointer le mot. La procédure utilisée par ces élèves est nettement sémantique. Il déduisent le pluriel d'immeubles du sens du mot peuplée. En STS, les difficultés de marquage du pluriel se concentrent sur les mots adieux et instants. Là encore, ces formes sont inscrites dans des configurations syntaxiques qui ne permettent pas une identification rapide du nombre par la forme du déterminant à l'oral. En effet, adieux est précédé du déterminant leurs presque 
homophonique au singulier et au pluriel. La liaison a été faite à l'oral mais elle n'a pas été perçue par tous les enquêtés puisque ceux qui écrivent adieu sont nombreux à écrire leur. Le nom instants est intégré au syntagme ses tout derniers instants. Le déterminant est certes marqué en nombre à l'oral mais il est ici séparé du nom par un adverbe et un adjectif. En STS, on peut remarquer par ailleurs qu'un nom au singulier concentre un grande nombre d'ajouts de marques du pluriel. Il s'agit de soin dans le syntagme avec beaucoup de soin. Cette forme n'a malheureusement pas été traitée en entretien. On peut néanmoins supposer que la difficulté qu'elle représente est liée à l'expression de l'indénombrable dans un contexte sémantique où apparait l'idée de pluralité à travers le déterminant indéfini beaucoup de.

Notre corpus confirme donc l'inégale difficulté du marquage du pluriel et du singulier mais souligne également l'importance de prendre en compte l'ensemble des caractéristiques lexicales, sémantiques et syntaxiques pour évaluer la difficulté de l'application des marques de nombre dans un syntagme.

\subsection{Formes adjectivales}

Le tableau 4 présente l'ensemble des formes adjectivales des deux enquêtes classées des moins réussies aux plus réussies en ce qui concerne le marquage du nombre. Les formes présentant plus de $10 \%$ d'écart de réussite entre le mot entier et la marque de nombre, à nouveau repassées en gras, sont nombreuses en troisième mais concernent majoritairement des formes au singulier. Les entretiens ont établi que le sens du participe passé bariolés était inconnu de nombreux élèves et que le participe passé perfectionnées était source de difficultés parce qu'il cumule une marque de genre et de nombre. En STS, ces formes sont moins nombreuses. Les mots règlementaires et éloigné concentrent de nombreuses erreurs d'accent. On retrouve donc au niveau des adjectifs la principales difficulté lexicale relevée en STS au niveau des noms.

Comme pour les noms, la marque de nombre est significativement plus souvent omise au pluriel qu'ajoutée au singulier. Les illustrations 3 et 4 montrent des phénomènes proches de ce qui a pu être observé pour les noms : le point le plus important est celui où se situent les élèves qui ne commettent aucune erreur. Ils sont cependant loin d'être majoritaires et les autres points sont répartis de manière très déséquilibrée vers l'axe des ordonnées, ce qui montre qu'ils commettent plus d'erreurs sur les marques de pluriel que sur les marques de singulier. On peut remarquer la présence d'effectifs importants sur les deux graphiques sur les points représentant les élèves ayant commis une ou plusieurs erreurs au pluriel et aucune au singulier. Cet élément montre que le marquage au pluriel des adjectifs demeure une difficulté importante chez des scripteurs avancés. 
Tableau 4: Formes adjectivales relevées dans la dictée de troisième $(\mathrm{N}=735)$ et la dictée de STS $(\mathrm{N}=178)$.

\begin{tabular}{|c|c|c|c|c|c|}
\hline $\begin{array}{l}\text { Formée dictée } \\
\text { de troisième }\end{array}$ & $\begin{array}{l}\text { Pourcentage } \\
\text { mot entier } \\
\text { réussi }\end{array}$ & $\begin{array}{l}\text { Pourcentage } \\
\text { marque du } \\
\text { nombre }\end{array}$ & $\begin{array}{l}\text { Forme dictée } \\
\text { STS }\end{array}$ & $\begin{array}{l}\text { Pourcentage mot } \\
\text { entier réussi }\end{array}$ & $\begin{array}{l}\text { Pourcentage réussite } \\
\text { marque du nombre }\end{array}$ \\
\hline microscopiques & $54,56 \%$ & $55,65 \%$ & parties & $55 \%$ & $69 \%$ \\
\hline perfectionnées & $40,14 \%$ & $59,05 \%$ & règlementaires & $58 \%$ & $69 \%$ \\
\hline verts & $57,14 \%$ & $59,05 \%$ & achevés & $78 \%$ & $82 \%$ \\
\hline bariolés & $38,37 \%$ & $59,32 \%$ & prête & $77 \%$ & $83 \%$ \\
\hline informé & $69,12 \%$ & $92,79 \%$ & derniers & $79 \%$ & $83 \%$ \\
\hline peuplée & $46,39 \%$ & $92,93 \%$ & jeunes & $97 \%$ & $97 \%$ \\
\hline vieil & $37,28 \%$ & 97,55 \% & international & $81 \%$ & $98 \%$ \\
\hline gentil & $87,21 \%$ & $99,18 \%$ & éloigné & $89 \%$ & $99 \%$ \\
\hline vieux & $83,13 \%$ & $100 \%$ & dernière & $94 \%$ & $99 \%$ \\
\hline extraordinaire & $83,67 \%$ & $100 \%$ & entier & $97 \%$ & $99 \%$ \\
\hline petit & $98,91 \%$ & $100 \%$ & & & \\
\hline $\begin{array}{l}\text { Total adjectifs } \\
\text { au singulier }\end{array}$ & $72,24 \%$ & $97,49 \%$ & $\begin{array}{l}\text { Total adjectifs } \\
\text { au singulier }\end{array}$ & $88 \%$ & $96 \%$ \\
\hline $\begin{array}{l}\text { Total adjectifs } \\
\text { au pluriel }\end{array}$ & $44,03 \%$ & $58,27 \%$ & $\begin{array}{l}\text { Total adjectifs } \\
\text { au pluriel }\end{array}$ & $73 \%$ & $76 \%$ \\
\hline Toutes formes & $63,29 \%$ & $83,23 \%$ & Toutes formes & $80 \%$ & $88 \%$ \\
\hline
\end{tabular}

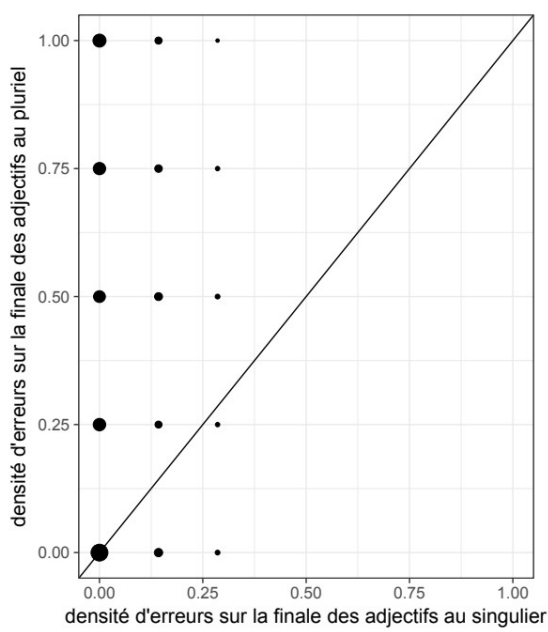

Illustration 3: Densité d'erreurs sur le marquage du nombre des adjectifs au singulier et au pluriel dans la dictée de troisième.

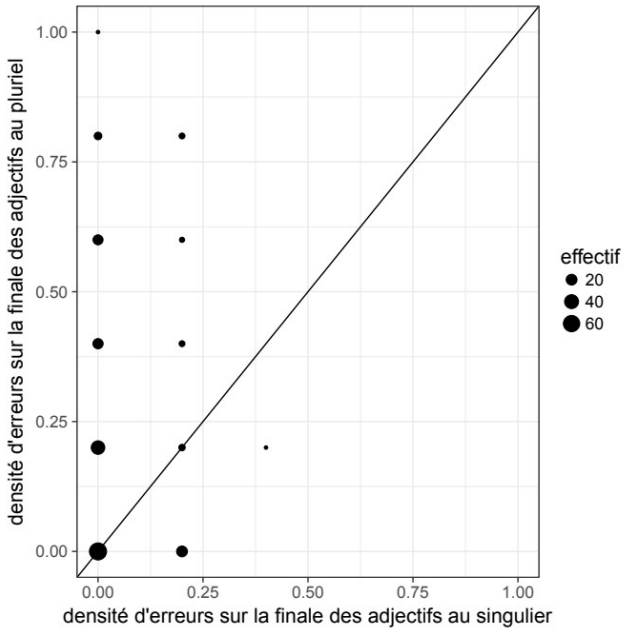

Illustration 4: Densité d'erreurs sur le marquage du nombre des adjectifs au singulier et au pluriel dans la dictée de STS.

Si on se reporte au tableau 4, on constate que la réussite du marquage du nombre au pluriel est assez homogène dans la dictée de troisième. En effet, les taux de réussite varient entre $55 \%$ et $60 \%$. Deux de ces formes ont été traitées dans l'entretien, verts dans ses yeux verts et bariolés dans peuplée d'immeubles bariolés. Pour verts, les commentaires recueillis montrent que les enquêtés qui s'interrogent sur le choix de la graphie de l'homophone [ver] oublient souvent de se poser la question de l'accord en genre et en nombre. Concernant bariolés, la 
réflexion semble souvent s'arrêter au choix d'une graphie en -é, là encore sans aller jusqu'à poser la question de l'accord en nombre. Dans un cas comme dans l'autre, les commentaires recueillis soulignent donc la difficulté que peut représenter le traitement de plusieurs questions différentes sur une même forme. Les deux autres adjectifs au pluriel sont des mot de trois syllabes. On peut donc émettre l'hypothèse que le traitement de leur difficulté lexicale a constitué un obstacle pour gérer les aspects morphosyntaxiques.

Le marquage du pluriel des adjectifs en STS apparait globalement mieux réussi mais plus hétérogène d'une forme à l'autre. La forme jeunes dans ces deux jeunes filles totalise $97 \%$ de marquage pertinent. Il est notable cependant que l'omission de la marque du pluriel est la seule erreur relevée sur jeunes. Tout se passe donc comme si l'orthographe lexicale du mot était suffisamment automatisée pour que le traitement de la marque de nombre soit optimal. On peut également remarquer que l'adjectif se situe juste après le numéral deux qui permet d'identifier facilement le nombre grammatical. Les deux formes recueillant moins de $70 \%$ de réussite de la marque du nombre sont parties et règlementaires. On a déjà pu constater que règlementaires, mot de quatre syllabes, posait de nombreux problèmes lexicaux aux élèves. La marque de pluriel ayant bien été identifiée par $96 \%$ des élèves sur le nom qui le précède, papiers, on peut supposer que c'est cette difficulté lexicale qui a fait obstacle à la réussite grammaticale. En ce qui concerne le participe passé adjectivé parties, $13 \%$ des élèves marquent le féminin mais pas le pluriel. Les entretiens permettent de confirmer que ces élèves ont bien identifié le donneur d'accord deux étudiantes mais se concentrent sur la marque de genre au détriment de la marque de nombre. Une proportion similaire d'élèves fait d'ailleurs le contraire et aboutit à la forme partis. Là encore, la faible réussite de la forme apparait donc liée au cumul de plusieurs traitements complexes nécessaires pour produire la forme normée.

\subsection{Comparaison entre la réussite des marques de nombre nominales et adjectivales}

Les difficultés observées pour marquer le nombre du nom et de l'adjectif chez ces scripteurs avancés sont donc structurellement similaires: elles sont plus marquées au pluriel qu'au singulier et varient en fonction des caractéristiques lexicales et syntaxiques de la forme. Néanmoins, la réussite du marquage du nombre est significativement meilleure sur les noms que sur les adjectifs dans les deux enquêtes.

Les illustrations 5 et 6 représentent la densité d'omission des marques du pluriel sur les noms en abscisse et sur les adjectifs en ordonnée dans les deux enquêtes. Il apparait que l'omission de la marque du pluriel est plus courante sur les adjectifs que sur les noms. En troisième, les élèves se répartissent de manière assez homogène dans la moitié du graphique représentant les élèves faisant autant ou plus d'erreurs sur les adjectifs que sur les noms. Cette répartition montre une grande diversité de profils, depuis les élèves ne rencontrant aucun problème de marquage du nombre à ceux en rencontrant systématiquement, en passant par ceux dont les difficultés se concentrent sur le marquage du pluriel de l'adjectif. En STS, le graphique est plus déséquilibré du côté du marquage du pluriel des adjectifs. Ceci indique que les difficultés de marquage du nombre chez ces enquêtés adultes se concentrent plus nettement sur le marquage du nombre de l'adjectif. 


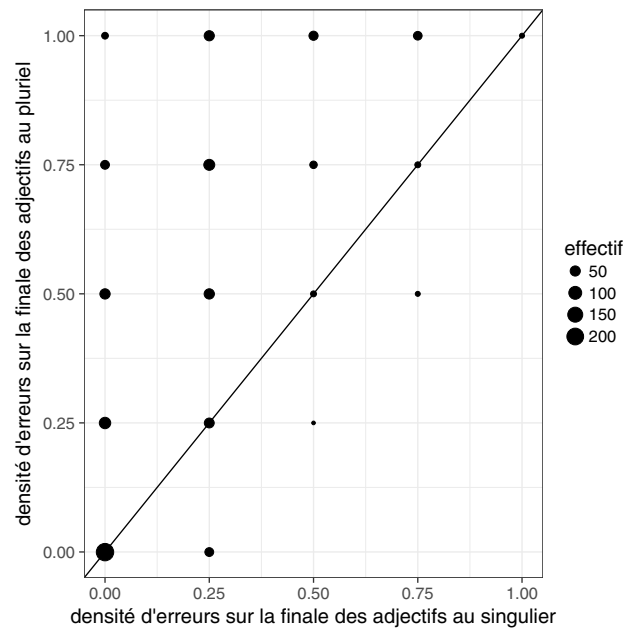

Illustration 6: Densité d'omission de la finale du pluriel sur les noms et sur les adjectifs dans la dictée de troisième.

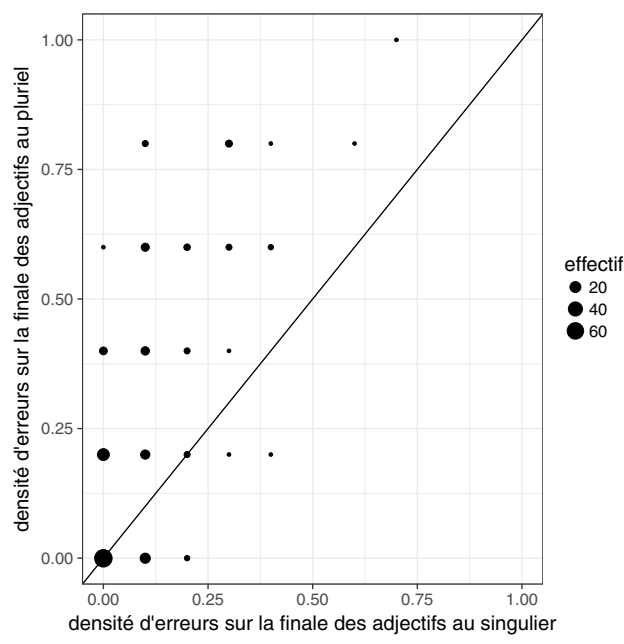

Illustration 5: Densité d'omission de la finale du pluriel sur les noms et sur les adjectifs dans la dictée de STS.

\section{Discussion}

Nos résultats confirment nos hypothèses. Chez des élèves avancés, scolarisés en fin de premier cycle de l'enseignement secondaire ou dans l'enseignement supérieur, marquer le nombre sur les formes nominales et adjectivales continue à poser problème, mais de façon non homogène suivant les configurations. L'omission de marque au pluriel est plus courante que l'ajout de marque au singulier. Ce phénomène est par ailleurs beaucoup plus marqué sur l'adjectif que sur le nom. Ces résultats sont conformes aux constats précédents de la recherche mais ils sont établis auprès d'une population peu ou pas testée de ce point de vue. Des élèves avancés dans leur scolarité, pour les STS très proches de leur entrée dans le monde professionnel, rencontrent encore des difficultés à traiter des questions d'orthographe grammaticale qui pourraient passer pour élémentaires, étudiées dès l'école primaire. D'un point de vue didactique, ce point amène nécessairement à s'interroger sur les modalités d'enseignement à l'école primaire et au collège d'une part et sur les possibilités de remédiation au lycée et dans l'enseignement supérieur d'autre part. Les résultats de notre double enquête nous amènent à la conclusion qu'il conviendrait d'envisager le pluriel nominal d'un point de vue morphosyntaxique, plutôt que de se limiter aux aspects morphologiques. Cela pourrait se faire en variant davantage les contextes des mots cibles et ce, dès l'école élémentaire.

L'analyse de la variabilité entre les formes étudiées peut fournir quelques pistes de réflexion. Tout d'abord l'importance du décalage entre la réussite des noms et celle des adjectifs invite à bien distinguer le fonctionnement de ces deux classes de mots dans l'enseignement des règles d'accord. Il apparait ensuite fondamental de prendre en compte l'imbrication entre des facteurs d'ordres différents dans les difficultés orthographiques qui se posent aux scripteurs. Nous avons ainsi pu constater que certaines configurations syntaxiques favorisaient l'apparition d'erreurs sur les marques de nombre. Il en va ainsi des structures qui séparent le nom du déterminant ou des déterminants dont la forme orale ne marque pas le pluriel. Mais certains éléments relevant de la difficulté lexicale du mot semblent influencer également la réussite de son marquage en nombre. Ainsi, chez les STS, la marque de pluriel est particulièrement bien réussie sur jeunes qui n'a posé par ailleurs aucun problème lexical. A contrario, la marque de nombre est souvent oubliée sur règlementaires qui en a posé beaucoup. Ces résultats sont cohérents avec ceux de Lusson (2013) qui a mis en évidence les 
effets de la fréquence, de la consistance orthographique et de certains éléments syntaxiques sur la réussite des accords en français. Il pourrait être intéressant de tester la réussite du marquage des mots en fonction de leur longueur et de leur degré d'opacité ou de consistance orthographique. Établir à quel point la complexité lexicale d'un mot peut faire obstacle au traitement de son orthographe grammaticale pourrait permettre de mieux accompagner les élèves dans leur apprentissage du marquage du nombre dans les groupes nominaux. Un outil facile d'emploi comme EOLE (Pothier et Pothier, 2002) pourrait être utilisé afin de sélectionner les mots cibles en fonction de leur difficulté lexicale.

Sur les adjectifs, la présence concomitante de la marque du pluriel et de celle du féminin fait partie des facteurs de complexification. Nos données se prêtaient plus difficilement à l'étude systématique de la question du marquage en genre. En effet, sur les noms, il s'agit d'une question lexicale et la présence de formes épicènes diminuait le nombre d'items sur lesquels pouvaient porter l'étude en ce qui concerne les adjectifs. Pour autant, nos données soulèvent des problèmes qui incitent à creuser la question du marquage en genre, par ailleurs peu étudiée (Cogis et Brissaud, 2019). Premièrement, on peut se demander si le fait que l'ensemble des noms et une partie des adjectifs ne portent pas de marque grammaticale du genre est compris par tous les enquêtés. L'ajout d'un $e$ au nom cité par près de $14 \%$ des élèves peut faire penser que ceux-ci pensent devoir marquer le genre du nom comme ils marquent celui de l'adjectif. Nos données ne permettent pas de vérifier cette hypothèse, mais celle-ci nous semblerait intéressante à explorer. Deuxièmement, les formes adjectivales les moins réussies dans les deux enquêtes sont des formes marquées en féminin. Il semblerait donc important de concevoir des enquêtes permettant une exploration plus systématique du marquage du féminin. D'un point de vue didactique, ces observations invitent à présenter le marquage en genre en distinguant nettement son marquage nominal et adjectival et en prenant réellement en compte l'importance des adjectifs épicènes. De fait, la marque - $e$ est beaucoup moins caractéristique du féminin nominal et adjectival que la marque $-s$ ne l'est du pluriel (Fayol et Brissaud, 2018). Ce phénomène complique l'analyse statistique du traitement du féminin mais explique peut-être également certaines difficultés de traitement par les scripteurs.Le double marquage du nombre et du féminin souligne par ailleurs la difficulté que représente pour certains scripteurs la prise en compte de plusieurs niveaux d'analyse différents sur une même forme.

\section{Références bibliographiques}

Andreu, C., et Steinmetz, C. (2016). Les performances en orthographe des élèves en fin d'école primaire (1987-2007-2015). Consulté à l'adresse http://www.education.gouv.fr/cid23433/les-performances-enorthographe-des-eleves-en-fin-d-ecole-primaire-1987-2007-2015.html

Blondel, C., Brissaud, C. et Rinck, F. (2016). Description des pratiques orthographiques de scripteurs ordinaires et de scripteurs en difficulté avec l'écrit à partir de l'analyse d'une dictée. SHS Web of conference 27. DOI : $10.1051 /$ shsconf $/ 20162707003$

Brissaud, C. (2015). Quels enseignements tirer de quatre-vingt-dix-huit dictées de Troisième ? Du décalage entre prescription et acquisition des élèves. Le français aujourd'hui, 190, 61-72. DOI : $10.3917 /$ lfa.190.0061

Brissaud, C., Chevrot, J.-P. et Lefrançois, P. (2006). Les formes verbales homophones en /E/ entre 8 et 15 ans: Contraintes et conflits dans la construction des savoirs sur une difficulté orthographique. Langue française, 151, 74-93. DOI : 10.3406/lfr.2006.6775

Brissaud C., et Chevrot J.P. (2011). The late acquisition of a major difficulty of French inflectional orthography: The homophonic /E/ verbal endings. Writing Systems Research, 3(2), 129-144. DOI : $10.1093 /$ wsr/wsr003

Brissaud, C. et Fayol, M. (2018). Étude de la langue et production d'écrits. CNESCO- IFÉ. En ligne : http://www.cnesco.fr/fr/ecrire-et-rediger/apprentissage-de-lecrit/ 
Chervel, A. (2008). Histoire de l'enseignement du français du XVIIe au XXe siècle. Paris: Retz.

Cogis, D. (2005). Pour enseigner et apprendre l'orthographe nouveaux enjeux, pratiques nouvelles école, collège. Paris: Delagrave.

Cogis, D. (2007). L'orthographe grammaticale : une difficulté majeure. In Manesse, D. et Cogis, D. (dir.), Orthographe, à qui la faute? p. 97-136. Issy-les-Moulineaux : ESF éditeur.

Cogis, D. et Brissaud, C. (2019). À la poursuite des marques de genre. In Mortamet, C. (dir.), L'Orthographe, pratiques d'élèves, pratiques d'enseignants, représentations (p. 43-71). Mont-SaintAignan : Presses universitaires de Rouen et du Havre.

David, J., Guyon, O. et Brissaud, C. (2006). Apprendre à orthographier les verbes: Le cas de l'homophonie des finales en /E/. Langue française, 151, 109-126. DOI : 10.3406/psy.2001.29557

Dister, A. et Moreau, M.-L. (2019). Le poids de l'orthographe lexicale et de l'orthographe grammaticale dans les dictées. In Mortamet, C. (dir.), L'Orthographe, pratiques d'élèves, pratiques d'enseignants, représentations (p. 21-42). Mont-Saint-Aignan : Presses universitaires de Rouen et du Havre.

Fayol, M., et Got, C. (1991). Automatisme et contrôle dans la production écrite : les erreurs d'accord sujet verbe chez l'enfant et l'adulte. L'année psychologique. 91(2), 187-205. DOI : $10.3406 /$ psy. 1991.29453

Fayol, M. et Jaffré, J.-P. (2014). L'orthographe. Paris: Presses universitaires de France.

Fayol, M. et Pacton, S. (2006). L'accord du participe passé: Entre compétition de procédures et récupération en mémoire. Langue française, 151, 59-73. En ligne : https://www.persee.fr/doc/lfr 0023-8368_2006 num_151_3 6774

Largy, P. et Dédéyan, A. (2002). Automatisme en détection d'erreurs d'accord sujet-verbe: Étude chez l'enfant et l'adulte. L'année psychologique, 102 (2), 201-234. DOI : 10.3406/psy.2002.29589

Largy, P., Fayol, M. et Lemaire, P. (1996) The Homophone Effect in Written French: The Case of VerbNoun Inflection Errors. Language and Cognitive Processes, 11(3), 217-256. DOI : $\underline{10.1080 / 016909696387178}$

Lefrançois, P. (2009). Évolution de la conception du pluriel des noms, des adjectifs et des verbes chez les élèves du primaire. Repères [En ligne], 39. DOI : 10.4000/reperes. 846

Lusson, C. (2013). Influence des facteurs non syntaxiques sur l'accord en nombre : approche développementale. (Thèse de doctorat: Université de Nice Sophia Antipolis). En ligne : https://tel.archives-ouvertes.fr/tel-00949029/document

Manesse, D. et Cogis, D.(2007). Orthographe à qui la faute? Issy-les-Moulineaux (Hauts-de-Seine): ESF éditions.

Mout, T. (2013). L'orthographe du français: Usages et représentations d'adultes socio-différenciés: Approche pluridiciplinaire. (Thèse de doctorat : Université de Grenoble). En ligne : http://www.theses.fr/2013GRENL023/document

Pach, C., Jacquemin, D., Millet, A., Billiez, J. (1990). Le quotidien de l'orthographe ou l'orthographe ordinaire. In Lucci, V., et Millet, A. (dir.), L'Orthographe de tous les jours (p. 45-138). Paris : Honoré Champion.

Pothier, B. et Pothier, P. (2002).EOLE : échelle d'acquisition en orthographe lexicale pour l'école élémentaire du CP au CM2. Paris : Retz.

Totereau, C., Thevenin, M.-G, Fayol, M. (1997). The development of the understanding of number morphology in written french (p. 97-115). In Perfetti, C. A., Rieben, L., et Fayol, M. (Éd.), Learning to spell research, theory, and practice across languages. Mahwah, N.J.: Lawrence Erlbaum Associates.

Trosseille, B. et Brissaud, C (2015). Entretien : La place de la dictée dans les évaluations conduites par la DEPP. In Brissaud C., et Mortamet C. (dir.), La dictée, une pratique sociale emblématique. Glottopol, 26. En ligne : http://glottopol.univ-rouen.fr/numero_26.html 
Voiriot-Cordary, N.(2005). Acquisition et gestion de la morphologie verbale flexionnelle au français à l'entrée au lycée. (Thèse de doctorat : Université de Bourgogne).

\section{Annexe}

Pour un élève donné, on modélise les accords en nombre des noms comme des évènements aléatoires indépendants d'un mot à l'autre, mais avec une probabilité d'erreur qui ne dépend que du nombre du nom. Le nombre d'erreurs au singulier, noté $X$, et le nombre d'erreurs au pluriel, noté $Y$, sont donc des variables aléatoires binomiales indépendantes. Notre hypothèse $\mathrm{H}_{0}$ est l'égalité entre la probabilité d'erreur dans l'accord du nombre au singulier et celle du nombre au pluriel. La variable $X$ conditionnée par l'égalité $X+Y=k$ suit alors une loi hypergéométrique de paramètres $k, \frac{m}{m+n}$ et $(m+n)$, où $m$ est le nombre de formes au singulier et $n$ le nombre de formes au pluriel. Une version centrée et réduite est donnée par le quotient

$$
Z=\frac{(n X-m Y) \sqrt{m+n-1}}{\sqrt{m n(X+Y)(m+n-X-Y)}}
$$

On calcule donc la somme $S$ des variables $Z$ obtenues pour chaque élève, supposées indépendantes. Par théorème central limite, le quotient $S / \sqrt{N}$ suit approximativement une loi normale centrée réduite, donc la fonction de répartition de la loi normale permet de calculer une valeur $p$ pour notre hypothèse $\mathrm{H}_{0}$. 